\title{
Effect of Asymptomatic Transmission and Emergence Time on Multi-strain Viral Disease Severity
}

\author{
Amir Reza Alizad-Rahvar ( $\nabla$ alizad@ipm.ir ) \\ Institute for Research in Fundamental Sciences (IPM) \\ Mehdi Sadeghi \\ National Institute for Genetic Engineering and Biotechnology
}

\section{Research Article}

Keywords: asymptomatic, basic reproduction number, COVID-19, disease severity, dominant strain, emergence time, multi-strain virus

Posted Date: May 17th, 2021

DOl: https://doi.org/10.21203/rs.3.rs-513446/v1

License: (c) (i) This work is licensed under a Creative Commons Attribution 4.0 International License.

Read Full License 


\section{RESEARCH ARTICLE}

2

\section{Effect of asymptomatic transmission and}

Amir Reza Alizad-Rahvar ${ }^{1 *}$ and Mehdi Sadeghi ${ }^{2 *}$

\section{Abstract}

Background: In a viral epidemic, the emergence of a novel strain with increased transmissibility (larger value of basic reproduction number $R_{0}$ ) sparks the fear that the increase in transmissibility is likely to lead to an increase in disease severity. It is required to investigate if a new, more contagious strain will be necessarily dominant in the population and resulting in more disease severity.

Methods: The impact of the asymptomatic transmission and the emergence time of a more transmissible variant of a multi-strain viral disease on the disease prevalence, disease severity, and the dominant variant in an epidemic was investigated by a proposed 2-strain epidemic model.

Results: The simulation results showed that considering only $R_{0}$, is insufficient to predict the outcome of a new, more contagious strain in the population. A more transmissible strain with a high fraction of asymptomatic cases can substantially reduce the mortality rate. If the emergence time of the new strain is closer to the start of the epidemic, the new, more contagious variant has more chance to win the viral competition and be the dominant strain; otherwise, despite being more contagious, it cannot dominate previous strains.

Conclusions: Three factors of $R_{0}$, the fraction of asymptomatic transmission, and the emergence time of the new strain are required to correctly determine the prevalence, disease severity, and the winner of the viral competition.

Keywords: asymptomatic; basic reproduction number; COVID-19; disease severity; dominant strain; emergence time; multi-strain virus 
${ }^{1}$ Background 1

2 2

The emergence of the novel coronavirus strain in the UK, called SARS-CoV-2 VOC

202012/01 or B.1.1.7, was shocking because this novel variant could be up to about

$40 \%$ more transmissible than pre-existing

5 . 5

${ }_{6}$ creased transmissibility can add between 0.4 and 0.7 to the basic reproduction ${ }_{6}$

${ }_{7}$ number $\mathrm{R}_{0}$. This news sparked the fear that the increase in transmissibility is likely

to lead to a large increase in hospitalization, intensive care unit (ICU) admission

rate, and mortality. However, the studies about previous variants of SARS-CoV-2

showed that despite the rise of the lab-confirmed cases, the COVID-19 case fatal10 ity rate $(\mathrm{CFR})$ declined, i.e., more transmissibility did not necessarily cause more 11 severity [2,3]. Other studies in the UK and England showed that besides increasing the COVID-19 cases, the hospitalization rate, the ICU admission rate, and the CFR 13 declined $[4,5]$. The preliminary explanation was the predominant shift towards pos14 itivity in younger age groups who have a better outcome. However, the analysis of German COVID-19 data [6], which was reported by age categories, showed that the 16 COVID-19 CFR declined across all age groups [7]. Interestingly, the older groups drove the overall reduction in CFR.

19 The public health authorities need to pinpoint the cause of this decline in the fatal-19 2oity rate in order to decide how to react against the newly emerged viral strains. The20 21decision to fight blindly against a novel strain because of its increased transmissibil-21 22ity is not necessarily the most comprehensive and effective solution. We need to take22 23other factors along with the transmissibility into account in our decision-making. 23

24 The 24 The spread of COVID-19 is an iceberg with the invisible part of being the asymp${ }^{25}$ tomatic transmission. The percent of asymptomatic cases who never experience ${ }^{25}$ ${ }^{26}$ COVID-19 symptoms remains uncertain. From about $20 \%$ to $50 \%$ of infected peo- ${ }^{26}$ ${ }^{27}$ ple are reported to be asymptomatic $[8,9,10]$. In a study, $39 \%$ of children aged ${ }^{27}$ ${ }^{28}$ 6-13 years tested positive for COVID-19 with no symptoms [11]. Different stud- ${ }^{28}$ ${ }^{29}$ ies reported an insionificant difference in the upper respiratory viral load between ${ }^{29}$

30 symptomatic and asymptomatic cases $[8,12]$. Even a new study found that asymp${ }^{31}$ tomatic patients had higher SARS-CoV-2 viral loads than symptomatic cases [13]. ${ }^{32}$ Consequently, the asymptomatic infected people could play a significant driver role ${ }^{32}$ ${ }^{33}$ in the community spread of COVID-19. The results of a study demonstrated that ${ }^{33}$ 




5 In this study, we investigate the effect of the emergent viral strain on the total 5

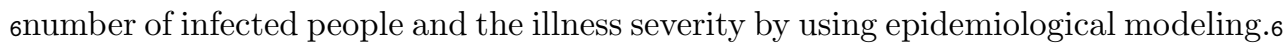
${ }_{7}$ We will show that in an epidemic situation, the emergence time of the new strain7 sand the relative $R_{0}$ of the primary and the emergent strains determine the winners gof the competition between two viral strains. Moreover, we will see that the diseaseg 10severity and the mortality rate can be significantly influenced by the emergence 10 11time and the fraction of asymptomatic infectious cases of the emergent strain. 11 $12+12$

$13+13$

$14+14$

$15+15$

$16-16$

$\begin{array}{ll}17 & 17\end{array}$

$18 \quad 18$

$19 \quad 19$

Methods 20

$21 \quad 21$

${ }^{22}$ For each viral strain, we use the extended version of the classic SEIRD epidemic ${ }^{22}$

${ }^{23}$ model, called the SEICARD model, consisting of susceptible $(\mathrm{S})$, exposed (in the ${ }^{23}$

${ }^{24}$ latent period) (E), symptomatic infected (I), critically infected (C), asymptomatic ${ }^{24}$

${ }^{25}$ infected $(\mathrm{A})$, recovered $(\mathrm{R})$, and dead $(\mathrm{D})$ people. By paralleling two SEICARD ${ }^{25}$

${ }^{26}$ models, we develop a 2-strain model, called 2-SEICARD, that describes the exis- ${ }^{26}$

${ }^{27}$ tence and competition of two viral variants in the population (Fig. 1). The index ${ }^{27}$

${ }^{28} s=1$ or 2 in $E_{s}, I_{s}, C_{s}, A_{s}, R_{s}$, and $D_{s}$ represents the infectious strain in each ${ }^{28}$

${ }^{29}$ group. It is assumed that the emergence time of the second strain is $T_{E}$ days after ${ }^{29}$

${ }^{30}$ the emergence time of the primary one, which is day 0. Moreover, we assume that ${ }^{30}$

${ }^{31}$ there is no viral superinfection, i.e., the reinfection or co-infection between variants ${ }^{31}$

${ }^{32}$ does not occur. In other words, the recovered individuals are cross-immunized and ${ }^{32}$

33 are immune to the new variants. 
1 The parameters of the 2-SEICARD model are explained in Table 1 . The ODE ${ }^{1}$ ${ }^{2}$ system of this 2 -strain model is given by

3

$6 \quad d E_{s} / d t=\left(\beta_{s}^{I} I_{s}+\beta_{s}^{C} C_{s}+\beta_{s}^{A} A_{s}\right) S / N-\varepsilon_{s} E_{s}$,

$7 \quad d I_{s} / d t=\left(1-P_{s}^{A}\right)\left(1-P_{s}^{C}\right) \varepsilon_{s} E_{s}-\gamma_{s}^{I} I_{s}$,

$8 \quad d C_{s} / d t=\left(1-P_{s}^{A}\right) P_{s}^{C} \varepsilon_{s} E_{s}-\left[\left(1-P_{s}^{D}\right) \gamma_{s}^{C}+P_{s}^{D} \gamma_{s}^{D}\right] C_{s}$,

$9 \quad d A_{s} / d t=P_{s}^{A} \varepsilon_{s} E_{s}-\gamma_{s}^{A} A_{s}$,

10

11

12

$$
d R_{s} / d t=\gamma_{s}^{I} I_{s}+\left(1-P_{s}^{D}\right) \gamma_{s}^{C} C_{s}+\gamma_{s}^{A} A_{s},
$$$$
d D_{s} / d t=P_{s}^{D} \gamma_{s}^{D} C_{s}
$$

13 14 15 16 for $t<T_{E}$.

17

${ }_{18}$ For each strain, the infection rates $\beta_{s}^{I}, \beta_{s}^{C}$, and $\beta_{s}^{A}$ denote the probability of ${ }_{18}$ ${ }_{19}$ transmitting disease from $I_{s}, C_{s}$, or $A_{s}$ to $S$, respectively. On the other hand, as ${ }_{19}$ ${ }_{20}$ Fig. 1 shows, the outcome of each exposed person $E_{s}$ could be $A_{s}, I_{s}, C_{s}$, and ${ }_{20}$ ${ }_{21} D_{s}$ with the probabilities of $P_{s}^{A},\left(1-P_{s}^{A}\right)\left(1-P_{s}^{C}\right),\left(1-P_{s}^{A}\right) P_{s}^{C}\left(1-P_{s}^{D}\right)$, and ${ }_{21}$ ${ }_{22}\left(1-P_{s}^{A}\right) P_{s}^{C} P_{s}^{D}$, respectively. By using the method of next-generation matrices [15], ${ }_{22}$ ${ }_{23}$ we can obtain the following expression for the $R_{0}$ of each strain

24

25

26

27

${ }^{28}$ where $R_{0}^{A(s)}, R_{0}^{I(s)}$, and $R_{0}^{C(s)}$ denote the reproduction number of each outcome, ${ }^{28}$ ${ }^{29}$ and $R_{0}^{(s)}$ is obtained by their weighted sum. The weight of each outcome is the ${ }^{29}$ ${ }^{30}$ probability of its occurrence.

30

31 The values of different parameters used in the 2-SEICARD model are listed in ${ }^{31}$

${ }^{32}$ Table 1. In our modeling, we assume a wild animal population in which there is no ${ }^{32}$ ${ }^{33}$ isolation and social policy or restriction. Hence, we consider that all $I_{s}, C_{s}$, and $A_{s}{ }^{33}$ 
${ }^{1}$ outcomes have the same probability of transmission; i.e., $\beta_{s}^{I}=\beta_{s}^{C}=\beta_{s}^{A}$. According ${ }^{1}$ ${ }^{2}$ to Eq. (2), by considering $\beta_{1}^{I}=\beta_{1}^{C}=\beta_{1}^{A}=0.2$ and the values of $0.13,0.2$, and $0.27^{2}$ ${ }^{3}$ for all $\beta$ values of strain 2 , we have $R_{0}^{(1)}=2$ and $R_{0}^{(2)}=1.3,2$, and 2.7 , respectively. ${ }^{3}$ 4 ${ }_{5}$ Results and Discussion

${ }_{6}$ In this section, we consider fixed parameters for the first strain in the 2-SEICARD 6 ${ }_{7}$ model; i.e., $R_{0}^{(1)}=2$ and $P_{1}^{A}=0.1$. Then, the emergent strain with different values ${ }_{7}$ ${ }_{8}$ of $R_{0}^{(2)}=1.3,2$, and 2.7 and $P_{2}^{A}=0.1,0.2$ and 0.4 emerges at day $T_{E}$, where ${ }_{8}$ ${ }_{9} 0 \leqslant T_{E} \leqslant 100$. In all the above scenarios, we study the effect of the emergent ${ }_{9}$ ${ }_{10}$ strain on the total number of infected cases and the mortality rate, as a measure of ${ }_{10}$ ${ }_{11}$ severity, from the beginning of the epidemic until we reach the endemic steady state ${ }_{11}$ ${ }_{12}$ The total number of infected cases is $N-S_{\infty}$, where $S_{\infty}$ denotes the number of ${ }_{12}$ ${ }_{13}$ susceptible cases that have not been infected at all when the disease has gone. The ${ }_{13}$ ${ }_{14}$ mortality rate is the total proportion of deaths in the population due to infection. ${ }_{14}$

${ }^{15}$ Effect of $R_{0}$ and $T_{E}$ on the total number of infections and the dominant strain 15 ${ }^{16}$ The simulation results show that the total number of infected cases does not vary ${ }^{16}$ ${ }^{17}$ with $P_{2}^{A}$ for the fixed values of $R_{0}^{(1)}$ and $R_{0}^{(2)}$. In other words, the values of $R_{0}^{(1)}$ and ${ }^{17}$ ${ }^{18} R_{0}^{(2)}$ determine the total number of infected individuals during the epidemic spread. ${ }^{18}$ ${ }^{19}$ Provided that $R_{0}^{(2)}<R_{0}^{(1)}$, the emergent strain does not have any chance to compete ${ }^{19}$ ${ }^{20}$ with the primary strain and would become extinct immediately (see Fig. 2(A1)). ${ }^{20}$ ${ }^{21}$ In the case of $R_{0}^{(2)}=R_{0}^{(1)}$, the total number of infected cases with two strains ${ }^{21}$ ${ }^{22}$ remains the same as that in the case of spreading only the primary strain in the ${ }^{22}$ ${ }^{23}$ population with the same value of the basic reproduction number. Moreover, Fig ${ }^{23}$ population with the same value of the basic reproduction number. Moreover, Fig.

${ }^{24}$ (A2) demonstrates that the total number of infected cases does not vary with the ${ }^{24}$ ${ }^{25}$ emergence time of the second strain, $T_{E}$. However, the later emergence of strain $2^{25}$ ${ }^{26}$ results in less proportion of infection with this strain in the population. In contrast, ${ }^{26}$ ${ }^{27}$ Fig. 2(A3) depicts that the emergence of a more contagious strain $\left(R_{0}^{(2)}>R_{0}^{(1)}\right)^{27}$ ${ }^{28}$ increases the total number of infected cases compared to the existence of only the ${ }^{28}$ ${ }^{29}$ primary strain. Furthermore, Fig. 2(A3) shows that the new, more contagious strain ${ }^{29}$ ${ }^{30}$ with larger value of $R_{0}$ does not necessarily dominate in the population. In ${ }^{30}$ ${ }^{31}$ words, $R_{0}$ alone does not determine which virus wins the viral competition. Indeed, ${ }^{31}$ ${ }^{32}$ besides the values of $R_{0}$, the emergence time $T_{E}$ also determines whether the new ${ }^{32}$ ${ }^{33}$ strain with more transmissibility dominates the primary one or not. The sooner ${ }^{33}$ 
${ }^{1}$ emergence of the new, more contagious variant can make it dominant; otherwise ${ }^{1}$

${ }^{2}$ the primary strain remains dominant in the population.

2

3

3

${ }_{4}$ Effect of emergent strain on mortality rate

4

${ }_{5}$ Our main question is that in the case of $R_{0}^{(2)}>R_{0}^{(1)}$, whether or not more infected ${ }_{5}$ ${ }_{6}$ cases increase similarly the mortality rate of the disease or not. To answer this ${ }_{6}$ ${ }_{7}$ question, consider the mortality rate in different circumstances in Figures $2\left(\mathrm{~B}_{-}{ }_{7}\right.$ $\left.{ }_{8} \mathrm{~B} 3\right)$. These figures show concurrently the effect of the fraction of asymptomatic ${ }_{8}$ ${ }_{9}$ individuals infected with strain $2, P_{2}^{A}$, and the emergence time of the second strain, 9 ${ }_{10} T_{E}$, on the mortality rate for $R_{0}^{(1)}=2$ and $R_{0}^{(2)}=1.3,2$, and 2.7. As we have ${ }_{10}$ ${ }_{11}$ discussed, if $R_{0}^{(2)}<R_{0}^{(1)}$, the emergent strain cannot compete with the primary ${ }_{11}$ ${ }_{12}$ strain, and hence, the mortality rate remains fixed for all values of $P_{2}^{A}$, equal to the ${ }_{12}$ ${ }_{13}$ mortality rate of the primary strain alone. Hence, in these figures, although blue ${ }_{13}$

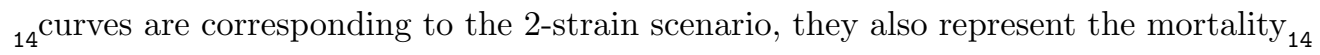
${ }_{15}$ rate of the primary strain alone.

15 ${ }_{16}$ As we expected, provided that $R_{0}^{(1)}=R_{0}^{(2)}$ and $P_{1}^{A}=P_{2}^{A}$, the mortality rate ${ }_{16}$ ${ }_{17}$ remains the same as that of the primary strain alone. On the other hand, as Fig. ${ }_{17}$ ${ }_{18} 2$ (B1) depicts, if both strains have a similar proportion of asymptomatic cases, i.e., ${ }_{18}$ ${ }_{19} P_{1}^{A}=P_{2}^{A}$, the mortality rate increases with the emergence of a more contagious ${ }_{19}$ ${ }_{20}$ strain $\left(R_{0}^{(2)}>R_{0}^{(1)}\right)$. In this case, the sooner that the new strain emerges, the more ${ }_{20}$ ${ }_{21}$ in the mortality rate increases. However, as Figures 2(B2-B3) show, the increase in ${ }_{21}$ ${ }_{22} P_{2}^{A}$ can reduce the mortality rate for $R_{0}^{(2)} \geq R_{0}^{(1)}$. For large values of $P_{2}^{A}$, although ${ }_{22}$ ${ }_{23}$ a more contagious strain emerges, it can make the mortality rate less than that be- ${ }_{23}$ ${ }_{24}$ fore the emergence (Fig. 2(B3)). Interestingly, for large values of $P_{2}^{A}$, the emergent ${ }_{24}$ ${ }_{25}$ strain with higher $R_{0}$ decreases the mortality rate more. In other words, more trans- ${ }_{25}$ ${ }_{26}$ missibility does not reflect necessarily more severity, and both $R_{0}^{(s)}$ and $P_{s}^{A}$ values $_{26}$ ${ }_{27}$ should be considered to correctly determine the effect of the more transmissible new $_{27}$ ${ }_{28}$ strain on the viral disease severity.

${ }^{29}$ Conclusion $\quad 29$

${ }^{30}$ In this study, we investigated the impact of the asymptomatic transmission and the

31 (1) 31

${ }^{32}$ disease severity, and the dominant variant in an epidemic. Our results demonstrated ${ }^{32}$

${ }^{33}$ that being an emergent strain with more transmissibility, i.e., having a larger basic ${ }^{33}$ 
${ }^{1}$ reproduction number $R_{0}$, compared to previous variants, does not necessarily lead ${ }^{1}$

${ }^{2}$ to more severity or mean that the new variant will dominate in the population. ${ }^{2}$

${ }^{3}$ Indeed, when a new strain with a larger basic reproduction number emerges, it will ${ }^{3}$

${ }^{4}$ increase the number of infected cases in the population. However, the creation of ${ }^{4}$

${ }^{5}$ more severe outcomes depends on the fraction of asymptomatic transmissions. If $\mathrm{a}^{5}$

${ }^{6}$ large proportion of infections, due to the new variant, do not show any symptom, ${ }^{6}$

${ }^{7}$ they can even reduce the mortality rate in the population. Moreover, provided that ${ }^{7}$

${ }^{8}$ the emergence time of the new strain is closer to the start of the epidemic, the ${ }^{8}$

${ }^{9}$ new, more contagious variant has more chance to win the viral competition and be ${ }^{9}$

${ }^{10}$ the dominant strain; otherwise, despite being more contagious, it cannot dominate ${ }^{10}$

${ }^{11}$ previous strains. 11

$12+12$

13Acknowledgements 13

${ }_{14}$ The authors would like to thank A. Kalirad for constructive discussion that contributed to the development of this 14 research.

15 Funding

16 No funding is available. $\quad 16$

$\begin{array}{ll}17 \text { Abbreviations } & 17\end{array}$

${ }_{18}$ CFR: Case fatality rate; COVID-19: Coronavirus disease 2019

${ }_{19}$ Availability of data and materials $\quad 19$

Not applicable.

$20 \quad 20$

Ethics approval and consent to participate
${ }^{21}$ Not required.

${ }^{22}$ Competing interests 22

${ }_{23}$ The authors declare that they have no competing interests. 23

${ }_{24}$ Consent for publication $\quad 24$

Not applicable.

Authors' contributions 25

${ }^{26}$ A.A. developed, analyzed, and simulated the methods, and wrote the manuscript. M.S. conceived the study and 26 ${ }_{27}$ guided the method development. All authors read and approved the final manuscript. 27

${ }_{28}$ Author details 28

${ }^{1}$ School of Biological Sciences, Institute for Research in Fundamental Sciences (IPM), Tehran, Iran. ${ }^{2}$ Department of

${ }^{29}$ Medical Genetics, National Institute for Genetic Engineering and Biotechnology, Tehran, Iran. 29

${ }^{30}$ References $\quad 30$

31 1. Davies NG, Barnard RC, Jarvis Cl, Kucharski AJ, Munday J, Pearson CAB, et al. Estimated transmissibility and 31 severity of novel SARS-CoV-2 Variant of Concern 202012/01 in England. medRxiv. 2020;.

32 2. SARS-CoV-2 Variants;. Available from: www. who.int/csr/don/31-december-2020-sars-cov2-variants/. 32

33 3. Why do COVID death rates seem to be falling?;. Available from: 33 www. nature.com/articles/d41586-020-03132-4. 
1 4. Howdon D, Oke J, Heneghan C. COVID-19: Declining Admissions to Intensive Care Units;. Available from:

2 Www.cebm.net/covid-19/covid-19-declining-admissions-to-intensive-care-units. 2

5. Howdon D, Heneghan C. The Declining Case Fatality Ratio in England;. Available from:

3 www.cebm.net/covid-19/the-declining-case-fatality-ratio-in-england. 3

4. RKI COVID19 data hub;. Available from: https:

//npgeo-corona-npgeo-de.hub.arcgis.com/datasets/dd4580c810204019a7b8eb3e0b329dd6_0/data.

1

2

5 7. Oke J, Howdon D, Heneghan C. Declining COVID-19 Case Fatality Rates across all ages: analysis of German 5 data;. Available from: https://www.cebm.net/covid-19/ declining-covid-19-case-fatality-rates-across-all-ages-analysis-of-german-data.

7 8. Ra SH, Lim JS, Kim Gu, Kim MJ, Jung J, Kim SH. Upper respiratory viral load in asymptomatic individuals 7 and mildly symptomatic patients with SARS-CoV-2 infection. Thorax. 2021;76(1):61-63.

8 9. Gudbjartsson DF, Helgason A, Jonsson H, Magnusson OT, Melsted P, Norddahl GL, et al. Spread of 8

$9 \quad$ SARS-CoV-2 in the Icelandic Population. New England Journal of Medicine. 2020;382(24):2302-2315. 9

10. Byambasuren O, Cardona M, Bell K, Clark J, McLaws ML, Glasziou P. Estimating the extent of asymptomatic

10 COVID-19 and its potential for community transmission: Systematic review and meta-analysis. Journal of the 10

11 Association of Medical Microbiology and Infectious Disease Canada. 2020;5:223-234.

11. Hurst JH, Heston SM, Chambers HN, Cunningham HM, Price MJ, Suarez L, et al. Severe Acute Respiratory

12 Syndrome Coronavirus 2 Infections Among Children in the Biospecimens from Respiratory Virus-Exposed Kids 12 (BRAVE Kids) Study. Clinical Infectious Diseases. 2020 11;.

13 12 . Cereda D, Tirani M, Rovida F, Demicheli V, Ajelli M, Poletti P, et al. The early phase of the COVID-19 13

14 outbreak in Lombardy, Italy. arXiv. 2020;Available from: https://arxiv.org/abs/2003.09320. 14

13. Hasanoglu I, Korukluoglu G, Asilturk D, Cosgun Y, Kalem AK, Altas AB, et al. Higher viral loads in

15 asymptomatic COVID-19 patients might be the invisible part of the iceberg. Infection. 2020 Nov;.

16 ${ }^{14}$. Fraser C, Riley S, Anderson RM, Ferguson NM. Factors that make an infectious disease outbreak controllable. 16 Proceedings of the National Academy of Sciences. 2004;101(16):6146-6151.

1715. Diekmann O, Heesterbeek JA, Roberts MG. The construction of next-generation matrices for compartmental 17 18 epidemic models. J R Soc Interface. 2010 Jun;7(47):873-885.

Figures

19

$212 \begin{aligned} & \text { Figure } 1 \text { Schematic of the 2-SEICARD model. For simplicity, the lower branch, corresponding to } \\ & 21 \\ & \text { the second strain, is not depicted, and it is denoted by } E I C A R D_{2} \text {. The } E I C A R D_{2} \text { branch } \\ & \text { appears in the model for } t \geqslant T_{E} \text {. }\end{aligned}$

24

25

26

27

27

28

8

$29 \quad R_{0}^{(1)}<R_{0}^{(2)}$ (A3). Also, these figures demonstrate that the emergent time of the new strain ( $\left.T_{E}\right)$

should be considered to determine the winner of the viral competition. The legends of all figures are the same as those in Fig. A1. B1-B3: For $R_{0}^{(1)}=2$ and $P_{1}^{A}=0.1$, these figures show the

31 effect of increase in the fraction of asymptomatic cases of the emergent strain $\left(P_{2}^{A}=0.1\right.$ (B1), 0.2 (B2), and 0.4 (B3)), on the mortality rate for different levels of transmissibility of the new variant $\left(R_{0}^{(2)}=1.3,2\right.$, and 2.7$)$. The legends of all figures are the same as those in Fig. B1.
24 33 


\section{${ }^{1}$ Tables}

${ }^{2}$ Table 1 Explanation of the symbols of the 2-SEICARD model.

\begin{tabular}{|c|c|c|c|}
\hline 3 & Symbol & Explanation & Value \\
\hline \multirow[b]{2}{*}{$4^{-}$} & $s$ & Strain number & 1,2 \\
\hline & $S$ & Susceptible individuals & \\
\hline 5 & $E_{s}$ & Exposed to strain $s$ and still in the latent period & 5 \\
\hline \multirow[b]{2}{*}{6} & $I_{s}$ & Symptomatic individuals infected with strain $s$ & \\
\hline & $C_{s}$ & Critically infectious individuals infected with strain $s$ & 6 \\
\hline 7 & $A_{s}$ & Asymptomatic individuals infected with strain $s$ & 7 \\
\hline \multirow{2}{*}{8} & $R_{s}$ & Individuals recovered from strain $s^{\prime}$ infection & \\
\hline & $D_{s}$ & Dead individuals infected with strain $s$ & 8 \\
\hline 9 & $N$ & Total number of individuals & 10000 \\
\hline 10 & $P_{s}^{A}$ & Fraction of asymptomatic individuals infected with strain $s$ & $\begin{array}{l}0.1(s=1) \\
0.10,0.2,0.4(s=2)\end{array}$ \\
\hline 11 & $P_{s}^{C}$ & $\begin{array}{l}\text { Fraction of symptomatic individuals who are critically } \\
\text { infected with strain } s\end{array}$ & 0.1 \\
\hline 13 & $P_{s}^{D}$ & $\begin{array}{l}\text { Fraction of critically infected individuals who die } \\
\text { from infection with strain } s\end{array}$ & $\begin{array}{c}12 \\
0.05 \\
13\end{array}$ \\
\hline 14 & $\beta_{s}^{I}, \beta_{s}^{C}, \beta_{s}^{A}$ & Infection rate of different outcomes of strain $s$ & $\begin{array}{l}0.2(s=1) \\
0.13,0.2,0.27(s=2)\end{array}$ \\
\hline 15 & $1 / \varepsilon_{s}$ & Average incubation period of strain $s$ & 5 1ckays \\
\hline & $1 / \gamma_{s}^{I}, 1 / \gamma_{s}^{C}, 1 / \gamma_{s}^{A}, 1 / \gamma_{s}^{D}$ & Average infection period of different outcomes of strain $s$ & 10 days \\
\hline & $T_{E}$ & Emergence time of strain 2 & $0 \leq T_{E} \leq 100$ day \\
\hline
\end{tabular}


Figures

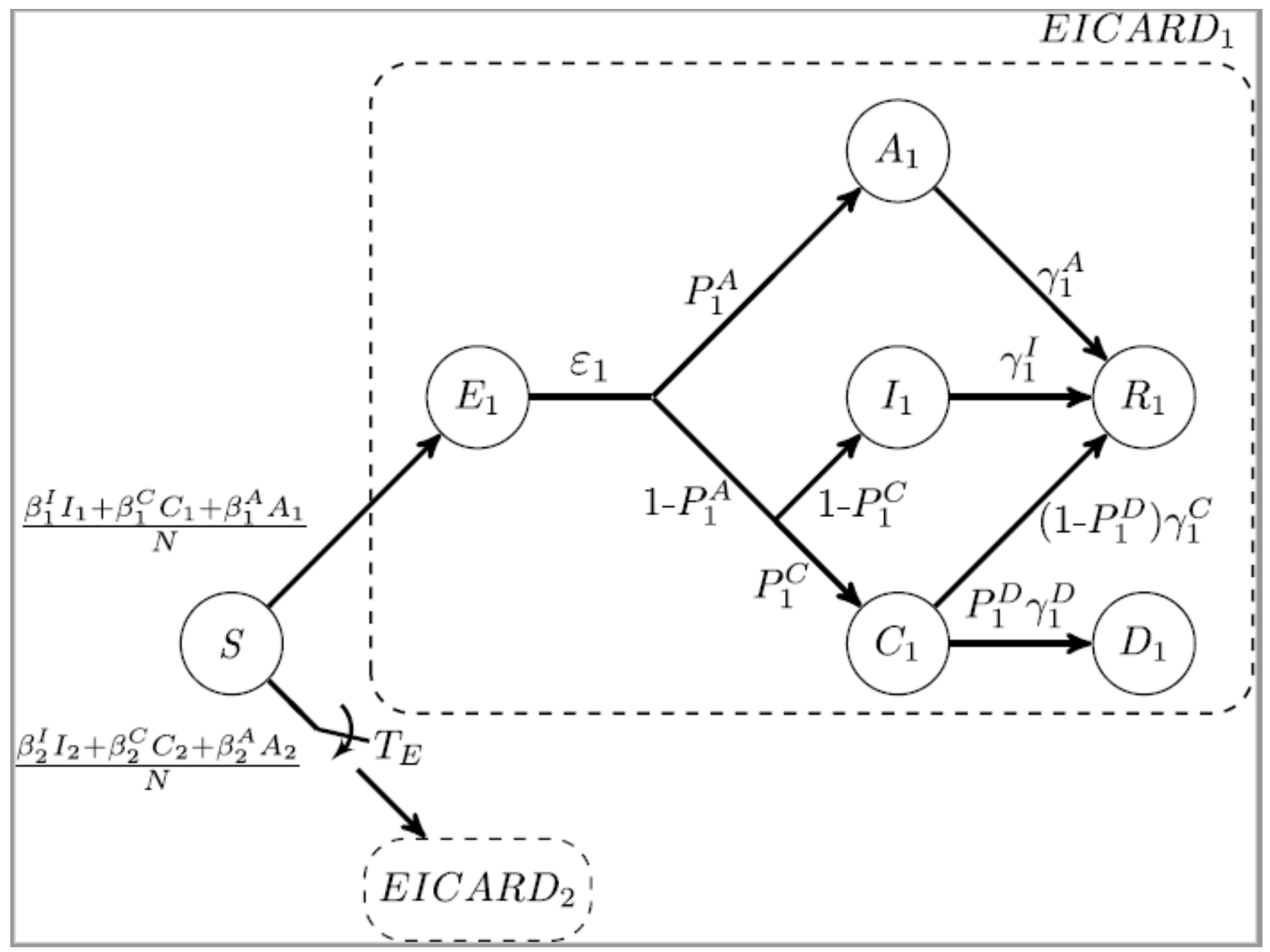

Figure 1

See the Manuscript Files section for the complete figure caption. 



Figure 2

See the Manuscript Files section for the complete figure caption. 